\title{
Multilevel regulation of RUVBL2 expression predicts poor prognosis in hepatocellular carcinoma
}

Tao Yan ${ }^{1 \dagger}$, Fang Liu' ${ }^{2 \dagger}$, Jiajia Gao ${ }^{2 \dagger}$, Haizhen Lu ${ }^{3}$, Jianqiang Cai ${ }^{4}$, Xiaohang Zhao ${ }^{2}$ and Yulin Sun ${ }^{2 *}$ (D)

\begin{abstract}
Background: Hepatocellular carcinoma (HCC) is the second-most lethal cancer worldwide with a complex pathogenesis. RuvB-like 2 (RUVBL2) was previously found to contribute to hepatocarcinogenesis. However, its expression, regulation and clinical significance have not been systematically evaluated in a large number of clinical samples.
\end{abstract}

Methods: Here, we performed a comprehensive analysis of RUVBL2 based on multiple datasets from 371 liver cancer patients of The Cancer Genome Atlas (TCGA) and on immunohistochemical staining in 153 subjects. In addition, the aberrant signaling pathways caused by RUVBL2 overexpression were investigated.

Results: We demonstrated that promoter hypomethylation, copy number gain, MYC amplification and CTNNB1 mutation were all responsible for RUVBL2 overexpression in HCC. High levels of RUVBL2 mRNA were associated with shorter recurrence-free survival time (RFS) but not overall survival time (OS). Furthermore, RUVBL2 protein was overexpressed in the nucleus and cytoplasm of HCC samples. Univariate and multivariate survival analyses showed that strong nuclear and cytoplasmic staining of RUVBL2 independently predicted worse OS and RFS with a 2.03-fold and a 1.71-fold increase in the hazard ratio, respectively. High levels of RUVBL2 promoted carcinogenesis through the heat shock protein 90 (HSP90)-Cell Division Cycle 37 (CDC37), AKT serine/threonine kinase (AKT) and mitogen-activated protein kinase (ERK/MAPK) pathways.

Conclusion: The deregulation of RUVBL2 in HCC is influenced at the genomic, epigenetic and transcriptional levels. Our findings highlight the potential roles of RUVBL2 as a promising prognostic marker as well as a therapeutic target for HCC.

Keywords: Liver cancer, Prognostic factor, RuvB-like 2, Regulation, Mechanism

\section{Background}

Liver cancer is the most commonly diagnosed cancer and the fourth leading cause of cancer death worldwide [1]. China accounts for approximately $60 \%$ of new liver cancer cases and deaths with a 5-year survival rate of $12 \%[2,3]$. Hepatocellular carcinoma (HCC) represents almost $90 \%$ of all primary liver cancer cases [4]. HCC is

\footnotetext{
*Correspondence: ylsun@cicams.ac.cn

${ }^{\dagger}$ Tao Yan, Fang Liu and Jiajia Gao contributed equally to this work

${ }^{2}$ State Key Laboratory of Molecular Oncology, National Cancer Center/

National Clinical Research Center for Cancer/Cancer Hospital, Chinese

Academy of Medical Sciences \& Peking Union Medical College, 17

Panjiayuan Nanli, Chaoyang District, Beijing 100021, China

Full list of author information is available at the end of the article
}

initiated by several risk factors, including chronic hepatitis $B$ virus $(\mathrm{HBV})$ and hepatitis $\mathrm{C}$ virus $(\mathrm{HCV})$ infections, alcohol abuse, autoimmune hepatitis, diabetes mellitus, obesity, and several metabolic diseases [5]. However, the molecular mechanisms of HCC remain only partially understood. The commonly involved pathways underlying hepatocarcinogenesis include telomere maintenance, WNT- $\beta$-catenin pathway, tumor protein 53 (TP53) signaling, oxidative stress signaling, epigenetic and chromatin remodeling, as well as AKT serine/threonine kinase (AKT)-mechanistic target of rapamycin kinase (mTOR)mitogen-activated protein kinase (MAPK/ERK) signaling, etc. [4]. 
Recently, RuvB-like 2 (RUVBL2) was found to interact with catenin beta 1 (CTNNB1), telomerase reverse transcriptase (TERT), MYC proto-oncogene (MYC), nuclear factor-kappa B1 (NFKB1), etc. to regulate the cancerrelated signaling pathways in HCC. RUVBL2 belongs to the conserved ATPases associated with various cellular activities (AAA+) protein subfamily, which is characterized by the presence of conserved Walker $A$ and $B$ motifs that are involved in ATP binding and hydrolysis [6, 7]. Due to its chaperone characteristics, this subfamily helps to assemble multiple complexes to participate in many biological functions, including those regulating nutrient sensing, transcription, chromatin remodeling, telomerase assembly, RNA metabolism, and DNA damage repair [8, 9]. For transcriptional regulation, RUVBL2 can activate MYC- and E2F transcription factor 1 (E2F1)-dependent transcription; however RUVBL2 represses CTNNB1, hypoxia-inducible factor 1-alpha (HIF1A), TP53, activating transcription factor 2 (ATF2), nuclear factor kappa light chain enhancer of activated B cells (NF-kB) and MYB proto-oncogene (MYB)-dependent transcription [10-15]. This pattern differs from that of its homologous partner RUVBL1 [8]. Thus, RUVBL2 may contribute to tumorigenesis and cancer development; indeed, RUVBL2 overexpression has been reported in $\mathrm{HCC}$, colorectal cancer, renal cell carcinoma, gastric cancer, breast cancer and salivary gland cancer [11, 16-20].

Silencing RUVBL2 in HCC cells reduced cell growth, increased apoptosis and induced cell senescence and migration; therefore, it is associated with poor prognosis and chemoresistance [16, 21-23]. In addition, RUVBL2 controlled glucose and lipid metabolism and contributed to the pathogenesis of insulin resistance and non-alcoholic fatty liver disease via mTOR and PI3KAKT pathways [24, 25]. However, the previous studies mainly investigated the mRNA expression characteristics of RUVBL2 in limited HCC samples using real-time reverse transcript-PCR, while its protein expression levels was detected in only 20 clinical samples by immunohistochemical staining $[16,23]$. In addition, the possible transcriptional and epigenetic regulation mechanism of RUVBL2 remains unclear. In this study, we analyzed the mRNA expression characteristics and expression regulation of RUVBL2 in HCC using multiple datasets from The Cancer Genome Atlas (TCGA) and investigated the function, clinical and prognostic significance of RUVBL2 protein using immunohistochemical staining and functional assays.

\section{Methods}

\section{TCGA data mining}

The RNA sequencing, somatic copy number alteration, DNA methylation data and clinical information from
371 patients with liver cancer were obtained from TCGA (https://tcga-data.nci.nih.gov). The samples contained 361 HCC, seven hepatocholangiocarcinoma (mixed), and three fibrolamellar carcinoma cases. Among them, $355 \mathrm{HCC}$ cases had detailed clinical and follow-up information. The median duration of patient follow-up was 20 months.

\section{Clinical samples}

Formalin-fixed paraffin embedded (FFPE) tissue samples were collected after approval from the Institutional Review Board of the Cancer Institute and Hospital of Chinese Academy of Medical Sciences (Beijing, China). All patients were diagnosed as HCC by two senior pathologists and had not received chemo/radiotherapy before surgical operation. A total of 153 HCC tumor samples and paired adjacent nontumor liver tissue samples were collected (143 male, 10 female; median age, $54 \pm 11$ SD; range 31-83 years) during the period from March 2004 to September 2008. Among them, 81.7\% (125/153) patients were HBsAg positive, whereas 8.5\% (13/153) patients were HCV positive. Additionally, 9.2\% $(14 / 153)$ of the cases had no histologic evidence of cirrhosis, whereas $43.1 \%(66 / 153), 24.2 \%$ (37/153) and $23.5 \%$ $(36 / 153)$ of cases showed mild, moderate and severe cirrhosis, respectively. Furthermore, 98.0\% (150/153) of cases were Child-Pugh Grade A, whereas 2.0\% (3/153) of them were Grade B. The median follow-up time of all patients was 62 months (range 7 months to 165 months).

\section{Immunohistochemistry}

The tissue slides were deparaffinized and rehydrated at room temperature, then immersed in methanol containing 3\% hydrogen peroxide for $10 \mathrm{~min}$ to block endogenous peroxidase. Heat-induced epitope retrieval was performed in a water bath for $30 \mathrm{~min}$ in an antigen retrieval solution (0.1 M sodium citrate buffer, $\mathrm{pH}$ 6.0). After washing, the sections were incubated overnight with anti-RUVBL2 antibody (1:80 dilution, Cat No. 10195-1-AP; ProteinTech Group Inc., Chicago, IL, USA) at $4{ }^{\circ} \mathrm{C}$. The staining was performed using the Prolink-1 Plus HRP rabbit polymer detection kit (Golden Bridge International Inc., Bothell, WA, USA) according to the manufacturer's instructions. The images were captured using Aperio ScanScope CS software (Vista, CA, USA).

The results were evaluated separately by two independent pathologists. The RUVBL2 staining intensity and area were quantified as described previously [26]. Briefly, RUVBL2 staining area was scored as follows: $0,<5 \%$ of the epithelial cells in the respective lesions; $1,5-25 \%$ of the epithelial cells; $2,26-50 \%$ of the epithelial cells; 3 , $51-75 \%$ of the epithelial cells; and $4, \geq 75 \%$ of the epithelial cells. The intensity was graded as follows: 0 , negative; 
$1+$, weak (yellow); $2+$, moderate (light brown); and 3+, strong (dark brown). A final score between 0 and 12 was achieved by multiplication of the extent of positivity and intensity. A staining index was used in which 0 was considered negative, $1-3$ was weak, and $\geq 4$ was considered strong expression.

\section{Transient transfection and Western blot analysis}

The human liver cancer cell lines HepG2 and Huh7 were purchased from the Institute of Biochemistry and Cell Biology of Chinese Academy of Sciences (Shanghai, China) and maintained in recommended media at $37^{\circ} \mathrm{C}$ with $5 \% \mathrm{CO}_{2}$. The cells were authenticated via Short Tandem Repeat profile, which was performed by Microread Corporation in Beijing, China.

Two specific siRNA duplexes targeting RUVBL2 mRNA (RefSeq\#: NM_006666.2) were designed and synthesized by GenePharma (Shanghai, China). The siRUVBL2-1 and siRUVBL2-2 sequences were 5'-CCGGUCGGGCAG UCCUUAU- $3^{\prime}$ and $5^{\prime}$-CCAUCGGCGUUCGCAUCA A-3', respectively. As a control, a scrambled sequence of 5'-UUCUCCGAACGUGUCACGU-3' was used. The siRNAs were transiently transfected into HepG2 and Huh7 cells with Lipofectamine 2000 (Invitrogen, MA, USA) according to the manufacturer's protocol.

Cells were lysed using a lysis buffer containing $50 \mathrm{mM}$ Tris-HCl (pH 7.4), 150 mM NaCl, 1\% NP-40, 0.1\% SDS, and protease inhibitor cocktail (Roche, Germany). Protein samples were loaded to SDS-PAGE gel and then transferred to polyvinylidene difluoride membranes. After blocking with $10 \%$ nonfat milk in PBS-T $(0.1 \%$ Tween-20), the membranes were incubated with the following antibodies: anti-RUVBL2, anti-ERK (antiMAPK1), anti-p-ERK, anti-AKT, anti-p-AKT, anti-pCDC37 (1:1000 dilution; Cell Signaling Technology, Danvers, MA, USA); anti-p-HSP90, anti-CDC37 (1:1000 dilution; Santa Cruz Biotechnology, Dallas, TX, USA); anti-HSP90 (1:1000 dilution; Abcam, Cambridge, UK); and anti- $\beta$-actin (1:5000 dilution; Sigma-Aldrich, St. Louis, MO, USA). Following intensive washing, the membranes were developed and visualized with the ImageQuant LAS4000 system (GE Healthcare, Chicago, IL, USA).

\section{Cellular proliferation and colony formation assays}

Huh7 and HepG2 cells were transfected with scrambled control or specific siRNA oligonucleotides against RUVBL2 and seeded at a density of 3000 per well in 96-well plates. Cell viability was measured by cell counting kit-8 (CCK-8) assay (Dojindo, Japan). The absorbance at $450 \mathrm{~nm}$ was measured using a microplate reader.

The colony formation assay was performed in 6-well plates in which 1000 cells were seeded per well and cultured for 2 weeks. Colonies were counted manually after staining with $0.5 \%$ crystal violet.

\section{Migration and invasion assay}

Twenty-four hours after transfection with siRUVBL2-1, siRUVBL2-2 or scrambled control, 10,000 HuH7 cells or 50,000 HepG2 cells were added to the upper chamber containing $200 \mu \mathrm{L}$ of serum-free medium; then, $600 \mu \mathrm{L}$ of complete medium containing $10 \%$ FBS was added into the lower chambers as a chemoattractant. After $24 \mathrm{~h}$ of incubation, the upper chambers were stained with $0.25 \%$ crystal violet. The cells that penetrated through the membrane were observed under microscope and manually counted within eight random $100 \times$ fields. Transwell invasion assays were performed in the same protocol as the migration assay with the exception that the inserts were precoated with $30 \mu \mathrm{g}$ of Matrigel (Corning Incorporated, NY, USA) in culture medium.

\section{Statistical analysis}

The Mann-Whitney U test, Wilcoxon signed-rank test or Kruskal-Wallis test was used to compare the Read per Million (RPM) values among two or multiple groups. In addition, the correlation coefficients of log2-transformed RPM values were calculated by Spearman's rank correlation. Chi-square test was used to compare qualitative data. The Kaplan-Meier method combined with log-rank analysis was used to determine the relationship between the levels of RUVBL2 and patient survival. Univariate and multivariate survival analyses were performed using the Cox regression model. $P$ values $<0.05$ were considered significant. All analyses were performed and visualized using GraphPad Prism 6.0 (GraphPad Software Inc., La Jolla, CA, USA).

\section{Results}

RUVBL2 mRNA was significantly upregulated in liver cancer tissues

According to the RNA sequencing data from TCGA, we first observed $R U V B L 2$ expression between primary tumor and paired adjacent noncancerous tissues $(\mathrm{n}=50)$. $R U V B L 2$ mRNA was significantly upregulated in tumor tissues (Fig. 1a, $P<0.0001$ ). Moreover, when the samples of tumor tissues were expanded to 371 cases, RUVBL2 mRNA remained at an approximately 1.3 -fold increase in HCC (Fig. 1b, $P<0.0001$ ).

The correlation analyses between the clinical features and the RUVBL2 mRNA levels in the tumors showed that RUVBL2 expression was associated with sex, race, drinking status and differentiation degree (Fig. 1b, c). The male and Asian patients with drinking habits had higher levels of RUVBL2 mRNA than the female and Caucasian patients without alcohol-related 

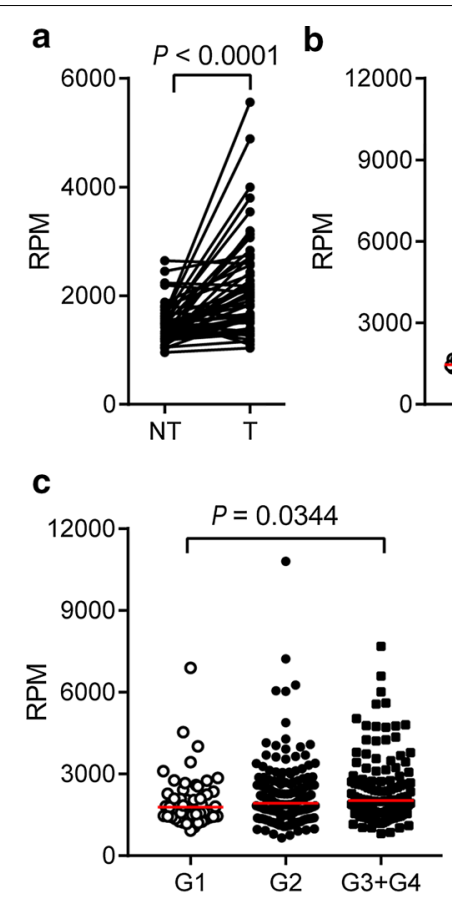

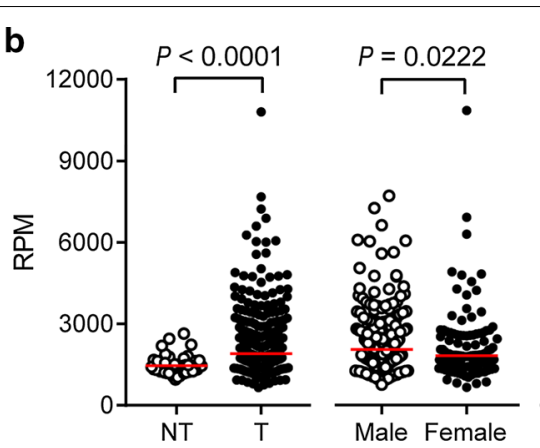

d

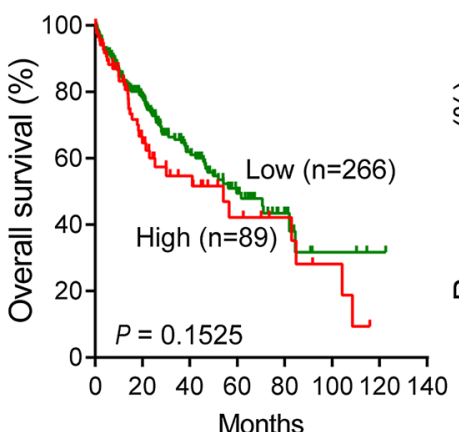

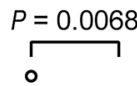

$P=0.0239$
$>$
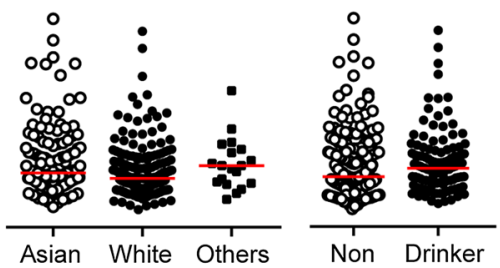

e

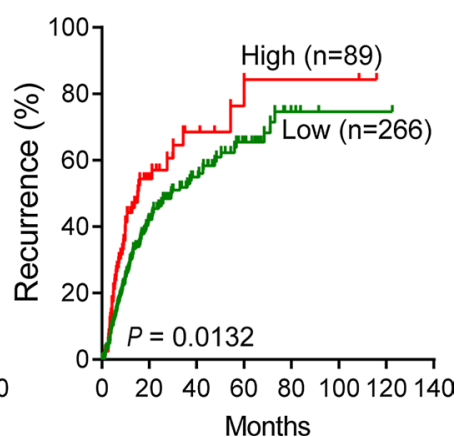

Fig. 1 The expression characteristics of RUVBL2 mRNA presented in TCGA liver cancer RNA sequencing dataset. a RUVBL2 mRNA expression in paired tumor and adjacent noncancerous tissues $(n=50)$. NT, nontumor tissues; T, tumor tissues; RPM, read per million. $\mathbf{b}$ Clinical significance of RUVBL2 mRNA expression in primary liver cancer tissues $(\mathrm{n}=371)$. White, Caucasian; Others, Black or African American and American Indian or Alaska Native. Non, nondrinkers. c RUVBL2 mRNA expression was associated with pathological differentiation degree in liver cancer according to the Edmondson grades (G1-G4). d, e Kaplan-Meier curves of overall survival (d) and recurrence-free survival (e) according to the RUVBL2 levels in tumor samples $(n=355)$. Log-rank test was performed

liver diseases (all, $P<0.05$ ). In addition, $R U V B L 2$ levels were higher in poorly differentiated tumors compared with those that were well differentiated $(P=0.0344)$. However, the correlation between RUVBL2 mRNA and other features, such as age, vascular invasion, ChildPugh classification, TNM staging, hepatic fibrosis degree, serum AFP levels and hepatic inflammation in adjacent liver tissue, was not observed.

Based on the quartile RPM values of RUVBL2 in tumor tissues, all 355 HCC cases with available followup information were divided into two groups: the highexpression group (top 25\%) and the low-expression group (bottom 75\%). Kaplan-Meier survival analysis with a log-rank test showed that RUVBL2 expression was not associated with the overall survival of the patients with liver cancer $(P=0.1525$; Fig. $1 d)$. However, there was a significant correlation between high RUVBL2 mRNA levels and a shorter recurrence-free survival time $(P=0.0132$; Fig. $1 \mathrm{e})$. The median relapse periods of high- and low-expression groups were 15.2 and 29.3 months, respectively.

\section{Aberrant transcriptional regulation of RUVBL2 mRNA in liver cancer}

To clarify why the RUVBL2 gene is overexpressed in liver cancer, we first observed the methylation of its promoter. As shown in Fig. 2a, the methylation level of $R U V B L 2$ was weakly inversely correlated with its mRNA levels, suggesting that promoter hypomethylation may participate in the overexpression of RUVBL2 gene (Pearson correlation coefficient $=-0.2354 ; P<0.0001$ ). When the copy number alterations of $R U V B L 2$ were compared with its expression levels, a weak positive correlation was observed (Spearman rank correlation coefficient $=0.3390, P<0.0001$ ). The gain of chromosome region near $R U V B L 2$ gene showed significantly higher mRNA expression than diploid and hemizygous deletion (Fig. 2b, $P<0.0001$ ).

The previous studies found that RUVBL2 physically interacts with a number of transcriptional factors (TFs), including CTNNB1, TATA-box binding protein (TBP), MYC, E2F1, ATF2 and HIF1A [6, 13, 27], and MYC transcriptionally activated RUVBL2 [28]. Given that $M Y C$ amplification and CTNNB1 mutation are the driver alterations in liver cancer, we thus detected whether these 
Table 1 Clinical significance of RUVBL2 staining in 153 HCC patients

\begin{tabular}{|c|c|c|c|c|c|c|c|c|}
\hline & \multicolumn{4}{|c|}{ Nuclear RUVBL2 } & \multicolumn{4}{|c|}{ Cytoplasmic RUVBL2 } \\
\hline & Negative & Weak & Strong & $P$ value & Negative & Weak & Strong & $P$ value \\
\hline Age (year) & & & & 0.6548 & & & & 0.3616 \\
\hline$<60$ & $55(53.4)$ & $32(31.1)$ & $16(15.5)$ & & $4(3.9)$ & $42(40.8)$ & $57(55.3)$ & \\
\hline$\geq 60$ & $23(46.0)$ & $17(34.0)$ & $10(20.0)$ & & $3(6.0)$ & $24(48.0)$ & $23(46.0)$ & \\
\hline Sex & & & & 0.6956 & & & & 0.6332 \\
\hline Male & $74(51.7)$ & $44(30.8)$ & $25(17.5)$ & & $7(4.9)$ & $60(42.0)$ & $76(53.1)$ & \\
\hline Female & $4(40.0)$ & $5(50.0)$ & $1(10.0)$ & & $0(0.0)$ & $6(60.0)$ & $4(40.0)$ & \\
\hline Family history & & & & 0.3719 & & & & 0.4666 \\
\hline Yes & $56(52.8)$ & $35(33.0)$ & $15(14.2)$ & & $3(2.8)$ & $45(42.5)$ & $58(54.7)$ & \\
\hline No & $22(46.8)$ & $14(29.8)$ & $11(23.4)$ & & $4(8.5)$ & $21(44.7)$ & $22(46.8)$ & \\
\hline Symptomatic presentation & & & & 0.1776 & & & & 0.9539 \\
\hline No & $54(56.8)$ & $27(28.4)$ & $14(14.7)$ & & $7(7.4)$ & $39(41.1)$ & $49(51.6)$ & \\
\hline Yes & $24(41.4)$ & $22(37.9)$ & $12(20.7)$ & & $0(0.0)$ & $27(46.6)$ & $31(53.4)$ & \\
\hline Drinking & & & & 0.0316 & & & & 0.3927 \\
\hline No & $59(52.7)$ & $30(26.8)$ & $23(20.5)$ & & $5(4.5)$ & $52(46.4)$ & $55(49.1)$ & \\
\hline Infrequent & $17(47.2)$ & $16(44.4)$ & $3(8.3)$ & & $2(5.6)$ & $11(30.6)$ & $23(63.9)$ & \\
\hline Frequent & $2(40.0)$ & $3(60.0)$ & $0(0.0)$ & & $0(0.0)$ & $3(60.0)$ & $2(40.0)$ & \\
\hline $\mathrm{HBsAg}$ & & & & 0.1774 & & & & 0.3722 \\
\hline Negative & $12(42.9)$ & $13(46.4)$ & $3(10.7)$ & & $0(0.0)$ & $14(50.0)$ & $14(50.0)$ & \\
\hline Positive & $66(52.8)$ & $36(28.8)$ & $23(18.4)$ & & $7(5.6)$ & $52(41.6)$ & $66(52.8)$ & \\
\hline Anti-HCV & & & & 0.9411 & & & & 0.4514 \\
\hline Negative & $72(51.4)$ & 44 (31.4) & $24(17.1)$ & & $7(5.0)$ & $58(41.4)$ & 75 (53.6) & \\
\hline Positive & $6(46.2)$ & $5(38.5)$ & $2(15.4)$ & & $0(0.0)$ & $8(61.5)$ & $5(38.5)$ & \\
\hline Cirrhosis & & & & 0.0416 & & & & 0.2321 \\
\hline $\mathrm{w} / \mathrm{o}+$ mild & $33(41.3)$ & $33(41.3)$ & $14(17.5)$ & & $3(3.8)$ & 31 (38.8) & $46(57.5)$ & \\
\hline Moderate & $23(62.2)$ & $7(18.9)$ & $7(18.9)$ & & $3(8.1)$ & $19(51.4)$ & $15(40.5)$ & \\
\hline Severe & $22(61.1)$ & $9(25.0)$ & $5(13.9)$ & & $1(2.8)$ & $16(44.4)$ & $19(52.8)$ & \\
\hline CEA (ng/ml) & & & & 0.8181 & & & & 0.8920 \\
\hline$\leq 5$ & $72(51.1)$ & $48(34.0)$ & $21(14.9)$ & & $5(3.5)$ & $63(44.7)$ & 73 (51.8) & \\
\hline$>5$ & $6(50.0)$ & $1(8.3)$ & $5(41.7)$ & & $2(16.7)$ & $3(25.0)$ & $7(58.3)$ & \\
\hline ALP & & & & 0.8388 & & & & 0.9196 \\
\hline Normal & 71 (51.1) & $46(33.1)$ & $22(15.8)$ & & $5(3.6)$ & $61(43.9)$ & $73(52.5)$ & \\
\hline Aberrant & $7(50.0)$ & $3(21.4)$ & $4(28.6)$ & & $2(14.3)$ & $5(35.7)$ & $7(50.0)$ & \\
\hline PT(a) (\%) & & & & 0.4977 & & & & 0.2135 \\
\hline$\geq 80$ & $51(54.3)$ & $26(27.7)$ & $17(18.1)$ & & $4(4.3)$ & $36(38.3)$ & $54(57.4)$ & \\
\hline$<80$ & $27(47.4)$ & 21 (36.8) & $9(15.8)$ & & $3(5.6)$ & 28 (30.6) & 26 (63.9) & \\
\hline $\operatorname{AFP}(\mathrm{ng} / \mathrm{ml})$ & & & & 0.2972 & & & & 0.1644 \\
\hline$\leq 20$ & $33(44.0)$ & $26(34.7)$ & 16 (21.3) & & $4(5.3)$ & $27(36.0)$ & 44 (58.7) & \\
\hline$>20$ & 41 (55.4) & $23(31.1)$ & $10(13.5)$ & & $3(4.1)$ & $37(50.0)$ & 34 (45.9) & \\
\hline $\operatorname{AFP}(\mathrm{ng} / \mathrm{ml})$ & & & & 0.7712 & & & & 0.5453 \\
\hline$\leq 400$ & $57(51.4)$ & 35 (31.5) & $19(17.1)$ & & $5(4.5)$ & $50(45.0)$ & $56(50.5)$ & \\
\hline$>400$ & $17(44.7)$ & 14 (36.8) & $7(18.4)$ & & $2(5.3)$ & $14(36.8)$ & $22(57.9)$ & \\
\hline Differentiation grade & & & & 0.5340 & & & & 0.0016 \\
\hline Well & $15(57.7)$ & $8(30.8)$ & $3(11.5)$ & & $5(19.2)$ & $14(53.8)$ & $7(26.9)$ & \\
\hline Moderate & $48(51.6)$ & 31 (33.3) & $14(15.1)$ & & $1(1.1)$ & $44(47.3)$ & $48(51.6)$ & \\
\hline Poor & $15(44.1)$ & $10(29.4)$ & $9(26.5)$ & & $1(2.9)$ & $8(23.5)$ & $25(73.5)$ & \\
\hline Tumor size (cm) & & & & 0.6152 & & & & 0.6543 \\
\hline$\leq 5$ & $53(52.5)$ & $33(32.7)$ & $15(14.9)$ & & $6(5.9)$ & $44(43.6)$ & $51(50.5)$ & \\
\hline$>5$ & $25(48.1)$ & $16(30.8)$ & $11(21.2)$ & & $1(1.9)$ & $22(42.3)$ & $29(55.8)$ & \\
\hline
\end{tabular}


Table 1 (continued)

\begin{tabular}{|c|c|c|c|c|c|c|c|c|}
\hline & \multicolumn{4}{|c|}{ Nuclear RUVBL2 } & \multicolumn{4}{|c|}{ Cytoplasmic RUVBL2 } \\
\hline & Negative & Weak & Strong & $P$ value & Negative & Weak & Strong & $P$ value \\
\hline Multinodules & & & & 0.9897 & & & & 0.9068 \\
\hline No & $62(50.8)$ & $39(32.0)$ & $21(17.2)$ & & $5(4.1)$ & $54(44.3)$ & $63(51.6)$ & \\
\hline Yes & $16(51.6)$ & $10(32.3)$ & $5(16.1)$ & & $2(6.5)$ & $12(38.7)$ & $17(54.8)$ & \\
\hline Tumor-infiltrating lymphocytes & & & & 0.6644 & & & & 0.8294 \\
\hline No & $57(50.4)$ & $35(31.1)$ & 21 (18.6) & & $6(5.3)$ & $49(43.4)$ & $58(51.3)$ & \\
\hline Yes & $21(52.5)$ & $14(35.0)$ & $5(20.7)$ & & $1(2.5)$ & $17(42.5)$ & $22(55.0)$ & \\
\hline Liver capsule invasion & & & & 0.7086 & & & & 0.7920 \\
\hline No & $48(52.7)$ & $27(29.7)$ & $16(17.6)$ & & $5(5.5)$ & $40(44.0)$ & $46(50.5)$ & \\
\hline Yes & $29(47.5)$ & $22(36.1)$ & $10(16.4)$ & & $2(3.3)$ & $26(42.6)$ & $33(54.1)$ & \\
\hline Carcinoma cell embolus & & & & 0.3656 & & & & 0.1208 \\
\hline No & $66(48.9)$ & $45(33.3)$ & $24(17.8)$ & & $7(5.2)$ & $61(45.2)$ & 67 (49.6) & \\
\hline Yes & $12(66.7)$ & $4(22.2)$ & $2(11.1)$ & & $0(0.0)$ & $5(27.8)$ & $13(72.2)$ & \\
\hline TNM staging & & & & 0.6785 & & & & 0.8315 \\
\hline 1 & $55(49.1)$ & $36(32.1)$ & $21(18.8)$ & & $4(3.6)$ & $51(45.5)$ & $57(50.9)$ & \\
\hline$\|$ & $11(52.4)$ & $8(38.1)$ & $2(9.5)$ & & $2(9.5)$ & $9(42.9)$ & $10(47.6)$ & \\
\hline III & $9(56.3)$ & $4(25.0)$ & $3(18.8)$ & & $0(0.0)$ & $6(37.5)$ & $10(62.5)$ & \\
\hline IV & $3(75.0)$ & $1(25.0)$ & $0(0.0)$ & & $1(25.0)$ & $0(0.0)$ & $3(75.0)$ & \\
\hline BCLC staging & & & & 0.4584 & & & & 0.3621 \\
\hline 0 & $4(66.7)$ & $0(0.0)$ & $2(33.3)$ & & $0(0.0)$ & $3(50.0)$ & $3(50.0)$ & \\
\hline 1 & $57(47.9)$ & $41(34.5)$ & 21 (17.6) & & $7(5.9)$ & $53(44.5)$ & 59 (49.6) & \\
\hline 2 & $12(54.5)$ & $7(31.8)$ & $3(13.6)$ & & $0(0.0)$ & $8(36.4)$ & 14 (63.6) & \\
\hline 3 & $5(83.3)$ & $1(16.7)$ & $0(0.0)$ & & $0(0.0)$ & $2(33.3)$ & $4(66.7)$ & \\
\hline
\end{tabular}

two TFs influenced $R U V B L 2$ expression. Intriguingly, the patients with $M Y C$ gain and amplification showed higher levels of RUVBL2 than those without this amplification (Fig. 2c-d). Furthermore, the individuals with CTNNB1 mutation had higher levels of RUVBL2 mRNA than those without the mutation (Fig. 2e, $P=0.0076$ ). However, there was an inverse correlation between the expression of RUVBL2 and CTNNB1 (Pearson correlation coefficient $=-0.2580 ; P<0.0001$; Fig. 2f). Taken together, RUVBL2 overexpression in liver cancer is caused by a variety of reasons, including promoter hypomethylation, chromosome gain and transcriptional regulation of TFs, etc.

\section{RUVBL2 protein was significantly overexpressed in HCC}

To further clarify the expression characteristics of RUVBL2 protein, an immunohistochemistry assay was performed in HCC tumor and adjacent noncancerous tissues $(n=153)$. In the adjacent noncancerous tissues, RUVBL2 was strongly stained in the bile duct epithelial cells (Fig. 3a), while the positive rate of hepatocytes was $80.0 \%(116 / 145)$, and the staining was mainly localized to cytoplasm (Fig. 3a, c and e). In these 116 samples, 65.5\% (76/116) and 35.5\% (40/116) showed weak and strong expression, respectively. Additionally, 35.2\% (51/145) of the cases showed positive nuclear staining of RUVBL2. The weak and strong nuclear staining comprised $84.3 \%$ (43/51) and $15.7 \%$ (8/53) of cases, respectively.

In contrast, positive cytoplasmic and nuclear immunostaining for RUVBL2 was observed in 95.4\% (146/153) and $46.0 \%(75 / 153)$ of the HCC tumors, respectively (Fig. $3 \mathrm{~b}, \mathrm{~d}$ and $\mathrm{f}$ ). In the positive cases that showed cytoplasmic staining for RUVBL2, 66 (45.2\%) and 80 (54.8\%) had weak and strong expression, respectively. Moreover, with regard to the nuclear staining, 49 (65.3\%) and $26(34.7 \%)$ had weak and strong expression, respectively. Collectively, apparent cytoplasmic and nuclear overexpression of RUVBL2 protein was found in HCC tissues (Chi-square test, $P<0.0001$ for the cytoplasm and $P=0.0037$ for the nucleus).

\section{Clinical significance of RUVBL2 protein in HCC}

The correlations between the clinicopathological characteristics of HCC patients and cytoplasmic and nuclear expression of RUVBL2 were subsequently analyzed (Table 1). Higher nuclear RUVBL2 levels were associated with drinking alcohol $(P=0.0316)$, whereas the patients with moderately and severe cirrhosis had lower levels of nuclear RUVBL2 $(P=0.0416)$. Higher cytoplasmic 

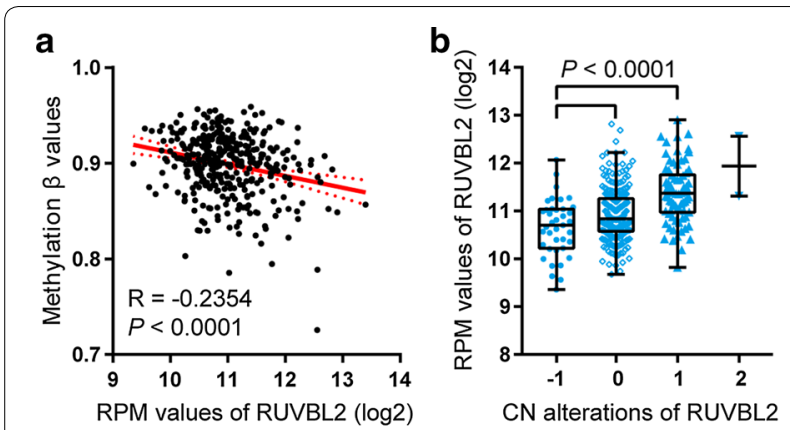

.
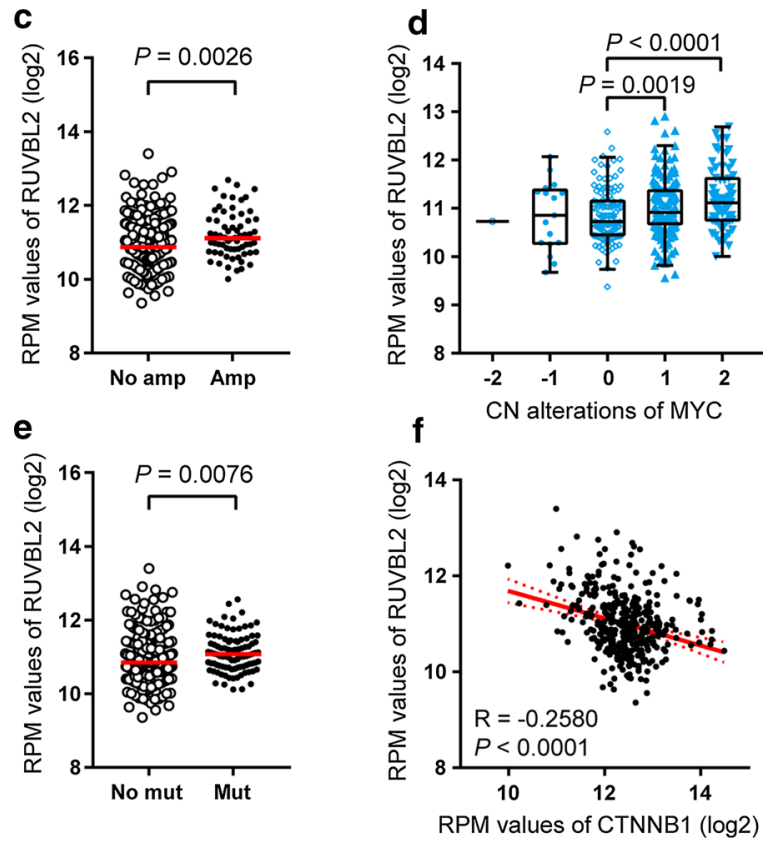

Fig. 2 DNA hypomethylation, gain/amplification, MYC amplification and driver mutation of CTNNB1 was responsible for the deregulation of RUVBL2. a RUVBL2 mRNA expression was inversely correlated with DNA methylation status in liver cancer based on the TCGA RNA-sequencing and DNA methylation $450 \mathrm{k}$ bead array datasets. Pearson correlation coefficients were calculated between the log2-transformed RPM values and methylation status of RUVBL2. $\mathbf{b}$ RUVBL2 mRNA expression showed gradient increase with the copy numbers of RUVBL2 gene. $\mathbf{c}$ The patients with MYC amplification had higher levels of RUVBL2 expression. No amp, no amplification; Amp, amplification. $\mathbf{d}$ RUVBL2 mRNA expression showed gradient increase with the copy numbers of MYC gene. For (b) and (d), values: $-2=$ homozygous deletion; $-1=$ hemizygous deletion; $0=$ neutral/no change; $1=$ gain; $2=$ high-level amplification. $\mathbf{e}$ The patients with CTNNB1 mutation had higher levels of RUVBL2 expression. No mut, no mutation; Mut, mutation. f RUVBL2 mRNA expression was inversely correlated with the levels of CTNNB1 in liver cancer. Pearson correlation coefficients were calculated between the log2-transformed RPM values of both genes

RUVBL2 levels were related to poor pathological differentiation $(P=0.0016)$.

However, there was no correlation between RUVBL2 protein and hepatitis virus infection, AFP, CEA, tumor size, multinodules, liver capsule invasion, carcinoma cell embolus, tumor-node-metastasis (TNM) stages or Barcelona Clinic Liver Cancer (BCLC) stages.

\section{Prognostic relevance of RUVBL2 protein in HCC}

To investigate the prognostic relevance of RUVBL2 protein in HCC, we performed Kaplan-Meier survival analysis with a log-rank test for nuclear and cytoplasmic expression levels. For nuclear staining, strong RUVBL2 expression was significantly associated with a shorter overall survival time $(P=0.0050$; Fig. $4 \mathrm{a})$. The median survival times of the strong and the negative/weak expression groups were 58 and 96 months, respectively. However, RUVBL2 expression was not associated with recurrence-free survival ( $P=0.1457$; Fig. $4 \mathrm{~b})$. For cytoplasmic staining, RUVBL2 expression had no significant influence on overall survival ( $P=0.0817$; Fig. 4c), whereas the patients with strong cytoplasmic RUVBL2 had significantly lower recurrence-free survival compared with those with negative and weak expression, with a median time to relapse of 26 months vs. 58 months $(P=0.0074$; Fig. 4d).

\section{RUVBL2 is an independent prognostic factor for HCC patients}

The subsequent univariate Cox regression analysis showed that patients with the strong nuclear RUVBL2 expression exhibited a 2.03-fold increase of a hazard ratio (HR) with a $95 \%$ confidence interval (CI) of 1.22 to 3.37 for overall survival (Table 2; $P=0.0064$ ), compared with the negative/weak expression group. Other significant risk factors for overall survival included differentiation grade $(\mathrm{HR}=1.30,95 \% \mathrm{CI} 1.02-1.66 ; P=0.0363)$, tumor size $(\mathrm{HR}=1.57,95 \% \mathrm{CI} 1.01-2.44 ; P=0.0459)$, carcinoma cell embolus $(\mathrm{HR}=2.19,95 \%$ CI $1.23-3.90$; $P=0.0080)$, TNM staging $(\mathrm{HR}=1.65$, 95\% CI 1.25 2.19; $P<0.0001)$ and BCLC staging $(\mathrm{HR}=2.45,95 \% \mathrm{CI}$ 1.49-4.04; $P<0.0001)$. As indicated by the multivariate analysis, strong nuclear RUVBL2 expression $(\mathrm{HR}=2.47$, 95\% CI 1.47-4.14; $P=0.0007)$, carcinoma cell embolus $(\mathrm{HR}=1.84,95 \%$ CI $1.01-3.36 ; P=0.0451)$ and BCLC staging $(\mathrm{HR}=2.32,95 \% \mathrm{CI} 1.59-3.40 ; P<0.0001)$ were independent prognostic factors for overall survival.

For the recurrent-free survival, in the univariate analysis, cytoplasmic RUVBL2 expression $(H R=1.71$, 95\% CI 1.44-2.56; $P=0.0088)$, differentiation grade $(\mathrm{HR}=1.27,95 \%$ CI $1.01-1.60 ; P=0.0409)$, tumor size $(\mathrm{HR}=1.69,95 \%$ CI $1.13-2.53 ; P=0.0107)$, carcinoma cell embolus $(\mathrm{HR}=2.66,95 \% \mathrm{CI} 1.56-4.53 ; P=0.0003)$, TNM staging $(\mathrm{HR}=1.54,95 \% \mathrm{CI} 1.18-2.00 ; P=0.0014)$ and BCLC staging $(\mathrm{HR}=2.10,95 \%$ CI $1.30-3.38$; $P=0.0023)$ were associated with the increased risk of 


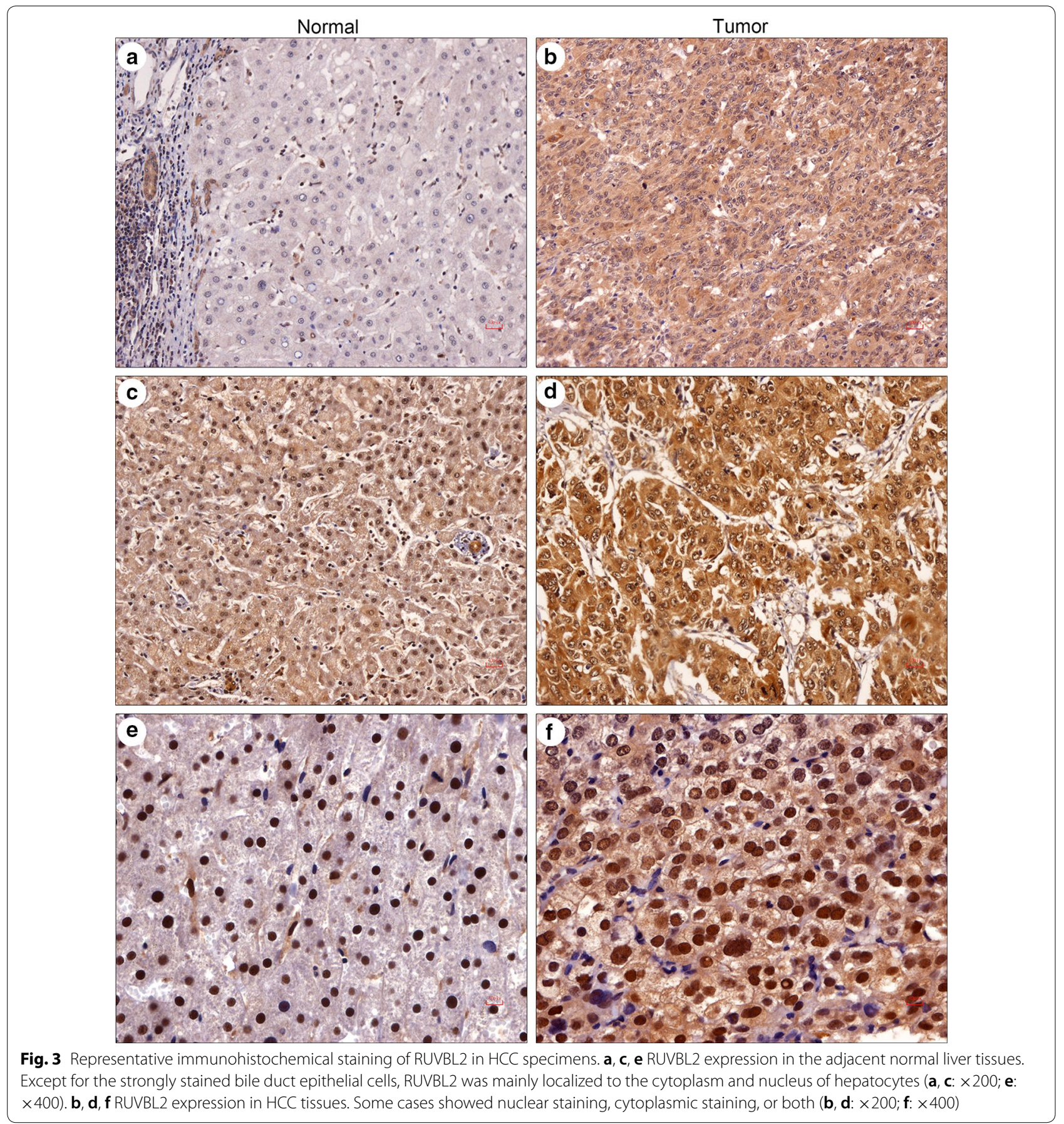

relapse (Table 2). Cytoplasmic RUVBL2 expression (HR $=1.56,95 \%$ CI $1.04-2.36 ; P=0.0336)$ was considered an independent recurrent factor, whereas tumor size $(\mathrm{HR}=1.45,95 \%$ CI $0.95-2.23 ; P=0.0887)$, carcinoma cell embolus $(\mathrm{HR}=1.75,95 \%$ CI $0.97-3.19$; $P=0.0652)$ and BCLC staging $(\mathrm{HR}=1.59,95 \% \mathrm{CI}$ $0.94-2.67 ; P=0.0811)$ showed marginal correlations.
RUVBL2 promotes cell malignant phenotypes through activating HSP90-CDC37, AKT and ERK pathways To investigate the potential function of RUVBL2 during hepatocarcinogenesis, we knocked down RUVBL2 expression in multiple cells. As shown in Fig. 5a-f, the short-term growth, long-term survival, migration and invasion abilities were markedly inhibited after the 

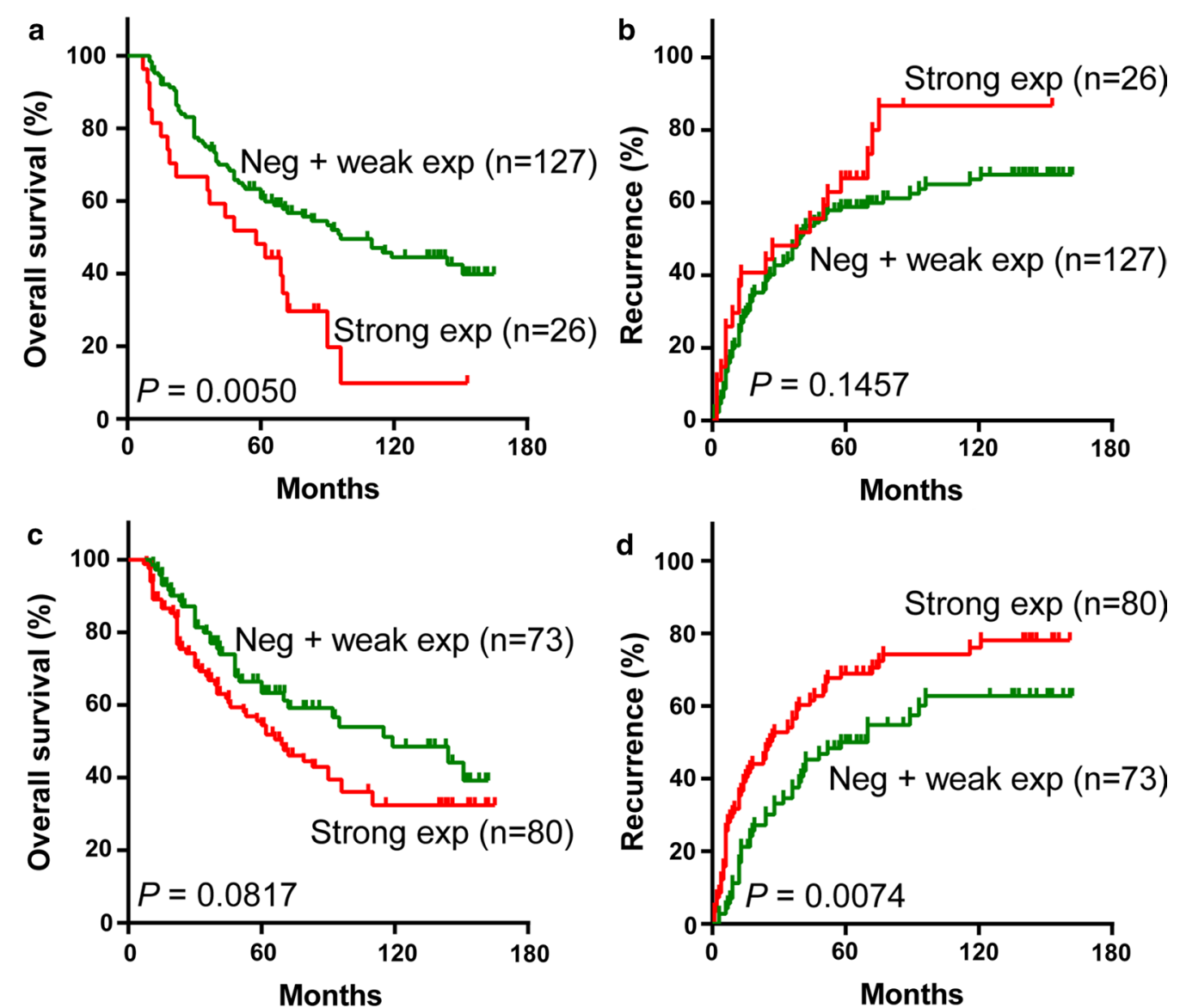

Fig. 4 Kaplan-Meier survival curves of HCC patients with nuclear and cytoplasmic RUVBL2 expression. a Overall survival analysis for RUVBL2 nuclear expression. b Recurrence-free survival analysis for RUVBL2 nuclear expression. c Overall survival analysis for RUVBL2 cytoplasmic expression. $\mathbf{d}$ Recurrence-free survival analysis for RUVBL2 cytoplasmic expression

transfection with specific siRNA against RUVBL2 compared with scrambled-transfected cells.

To gain insight into these mechanisms, we observed the expression levels of phosphorylated-CDC37 (p-CDC37), total CDC37, phosphorylated-HSP90 (p-HSP90), total HSP90, phosphorylated-ERK (p-ERK), total ERK, phosphorylated-AKT (p-AKT) and total AKT in RUVBL2 depleted cells. There was a significant trend of a decrease for p-CDC37, p-HSP90, p-AKT and p-ERK at $24 \mathrm{~h}$ after the transfection of RUVBL2 siRNA (Fig. $5 \mathrm{~g}$, h). Thus, it appears that RUVBL2 overexpression in HCC strengthens the proliferation, survival, migration and invasion of HCC cells through activating the HSP90-CDC37, AKT and ERK signaling pathways.

\section{Discussion}

In the present study, we found that $R U V B L 2$ mRNA was upregulated in HCC tissues, and promoter hypomethylation, copy number gain, $M Y C$ amplification and CTNNB1 mutation all contributed to its deregulation. High levels of RUVBL2 mRNA were associated with shorter recurrence-free survival time but not overall survival time. Furthermore, RUVBL2 protein that was localized at both the nucleus and cytoplasm was also overexpressed in HCC samples. Strong nuclear staining of RUVBL2 predicted worse overall survival, whereas strong intensity of cytoplasmic RUVBL2 was an independent unfavorable prognostic factor for recurrence-free survival.

Our results showed that high RUVBL2 mRNA expression was associated with poor differentiation of HCC tumor, which is in agreement with a previous report [16]. However, this finding is at odds with previous observations that RUVBL2 mRNA expression is significantly lower in HBV-related HCC [16]. We observed that male patients with an Asian background and a drinking habit had a higher expression of RUVBL2 mRNA. The difference might be a result of the different race and etiology composition of clinical samples. Actually, when the Asian and Caucasian people were separately analyzed, we found that RUVBL2 mRNA levels were indeed lower in 
Table 2 Univariate Cox regression analysis of overall and recurrence-free survival in 153 HCC patients

\begin{tabular}{|c|c|c|c|c|c|c|}
\hline \multirow[t]{2}{*}{ Variables } & \multicolumn{3}{|c|}{ Overall survival } & \multicolumn{3}{|c|}{ Relapse-free survival } \\
\hline & P-value & HR & $95 \% \mathrm{Cl}$ & P-value & HR & $95 \% \mathrm{Cl}$ \\
\hline Nuclear RUVBL2 (strong vs. Neg + weak) & 0.0064 & 2.03 & $1.22-3.37$ & 0.1522 & 1.42 & $0.88-2.31$ \\
\hline Cytoplasmic RUVBL2 (strong vs. Neg + weak) & 0.0862 & 1.47 & $0.95-2.27$ & 0.0088 & 1.71 & $1.44-2.56$ \\
\hline Age (>60 vs. $\leq 60)$ & 0.7896 & 1.06 & $0.68-1.66$ & 0.6266 & 1.11 & $0.73-1.67$ \\
\hline Sex (female vs. male) & 0.2357 & 0.54 & $0.20-1.49$ & 0.0713 & 0.40 & $0.15-1.08$ \\
\hline Family history (yes vs. no) & 0.0943 & 0.66 & $0.41-1.07$ & 0.0590 & 0.65 & $0.42-1.02$ \\
\hline Symptom presentation (yes vs. no) & 0.0864 & 1.46 & $0.95-2.26$ & 0.1258 & 1.36 & $0.92-2.03$ \\
\hline Drinking (yes vs. no) & 0.7888 & 0.94 & $0.62-1.44$ & 0.7493 & 0.94 & $0.65-1.37$ \\
\hline HBsAg (positive vs. negative) & 0.1842 & 0.71 & $0.42-1.18$ & 0.4802 & 0.84 & $0.51-1.37$ \\
\hline Anti-HCV (positive vs. negative) & 0.5942 & 1.23 & $0.57-2.68$ & 0.7104 & 1.15 & $0.56-2.37$ \\
\hline Cirrhosis (moderate + severe vs. w/o + mild) & 0.0545 & 1.52 & $0.99-2.34$ & 0.1645 & 1.32 & $1.89-1.96$ \\
\hline CEA (> 5 vs. $\leq 5)$ & 0.3999 & 1.40 & $0.64-3.04$ & 0.3377 & 1.40 & $0.70-2.78$ \\
\hline ALP (aberrant vs. normal) & 0.9062 & 0.96 & $0.50-1.84$ & 0.3606 & 1.29 & $0.75-2.24$ \\
\hline $\operatorname{AFP}(>20$ vs. $\leq 20)$ & 0.6581 & 1.10 & $0.71-1.70$ & 0.8061 & 1.05 & $0.71-1.56$ \\
\hline Differentiation grade (poor vs. well + moderate) & 0.0363 & 1.30 & $1.02-1.66$ & 0.0409 & 1.27 & $1.01-1.60$ \\
\hline Tumor size ( $>5 \mathrm{~cm}$ vs. $\leq 5 \mathrm{~cm}$ ) & 0.0459 & 1.57 & $1.01-2.44$ & 0.0107 & 1.69 & $1.13-2.53$ \\
\hline Multinodules (yes vs. no) & 0.0853 & 1.56 & $0.94-2.58$ & 0.1152 & 1.46 & $0.91-2.36$ \\
\hline Tumor-infiltrating lymphocytes (yes vs. no) & 0.7268 & 1.09 & $0.67-1.76$ & 0.4599 & 1.18 & $0.76-1.84$ \\
\hline Liver capsule invasion (yes vs. no) & 0.4503 & 0.84 & $0.54-1.31$ & 0.7892 & 0.95 & $0.63-1.42$ \\
\hline Carcinoma cell embolus (yes vs. no) & 0.0080 & 2.19 & $1.23-3.90$ & 0.0003 & 2.66 & $1.56-4.53$ \\
\hline TNM staging (III + IV vs. I+II) & $<0.0001$ & 1.65 & $1.25-2.19$ & 0.0014 & 1.54 & $1.18-2.00$ \\
\hline BCLC staging $(2+3$ vs. $0+1)$ & $<0.0001$ & 2.45 & $1.49-4.04$ & 0.0023 & 2.10 & $1.30-3.38$ \\
\hline
\end{tabular}

only HBV-infected Caucasian patients (Additional file 1: Figure S1), which is consistent with the previous finding [16]. This result suggested that the expression patterns and roles of RUVBL2 in hepatocarcinogenesis might be different in different ethnic groups.

Additionally, although RUVBL2 is a known transcription factor, the transcriptional regulation of $R U V B L 2$ itself remains unknown. Here, based on the multiple sequencing datasets from TCGA, we found that the methylation status and copy number alterations of RUVBL2 gene, MYC amplification as well as CTNNB1 mutation were all increased the mRNA expression of $R U V B L 2$. Interestingly, $R U V B L 2$ expression negatively correlated with CTNNB1 expression. Further analyses suggested that wild-type CTNNB1 repressed the transcription of RUVBL2, whereas CTNNB1 mutation might lose this function (Additional file 1: Figure S2). To our knowledge, this report is the first observation of transcriptional regulation of $R U V B L 2$ in tumors.

Intriguingly, most studies addressed that RUVBL2 is a nuclear protein. However, accumulating evidence has demonstrated the definite cytoplasmic staining of RUVBL2 protein, especially in malignant cells $[6,8,16$, 18]. We found that both cytoplasmic and nuclear staining of RUVBL2 was significantly increased in HCC. When cytoplasmic and nuclear staining were separately

\footnotetext{
(See figure on next page.)

Fig. 5 Knockdown of RUVBL2 expression inhibited cell proliferation and survival. a, d Cell proliferation was measured with CCK-8 assay at indicated times after the transfection of specific siRNA duplexes against RUVBL2 in HepG2 (a) and Huh7 (d) cells. The absorbance is shown as the mean \pm standard error for each day. $\mathbf{b}$, e The colony formation assay in HepG2 (b) and Huh7 (e) at $24 \mathrm{~h}$ after the transfection of specific siRNA duplexes against RUVBL2. Representative dishes are shown in the left panel, and quantitative colony numbers are compared in the right panel. c, $\mathbf{f}$ The migration and invasion assays (left panels) were performed in HepG2 (c) and Huh7 (f) at $24 \mathrm{~h}$ after the transfection of specific siRNA duplexes against RUVBL2. Cell migration and invasion capability is shown in the right panel by counting cells per field. For (a-f), Scr, scrambled negative control; siR-1, siRUVBL2-1; siR-2, siRUVBL2-2; ${ }^{*} P<<0.05 ;{ }^{* *} P<<0.01$; ${ }^{* * *} P<<0.001 ;{ }^{* * *} P<<0.0001$. g, $\mathbf{h}$ Western blot analysis of cell proliferation- and survival-associated signaling genes in HepG2 (g) and Huh7 (h) at $24 \mathrm{~h}$ post-transfection with specific siRNA duplexes against RUVBL2. Densitometry was performed to quantify each lane, and the ratio of each protein over the loading control $\beta$-actin is presented under each blot, with the ratio in the scramble group being the reference value
} 


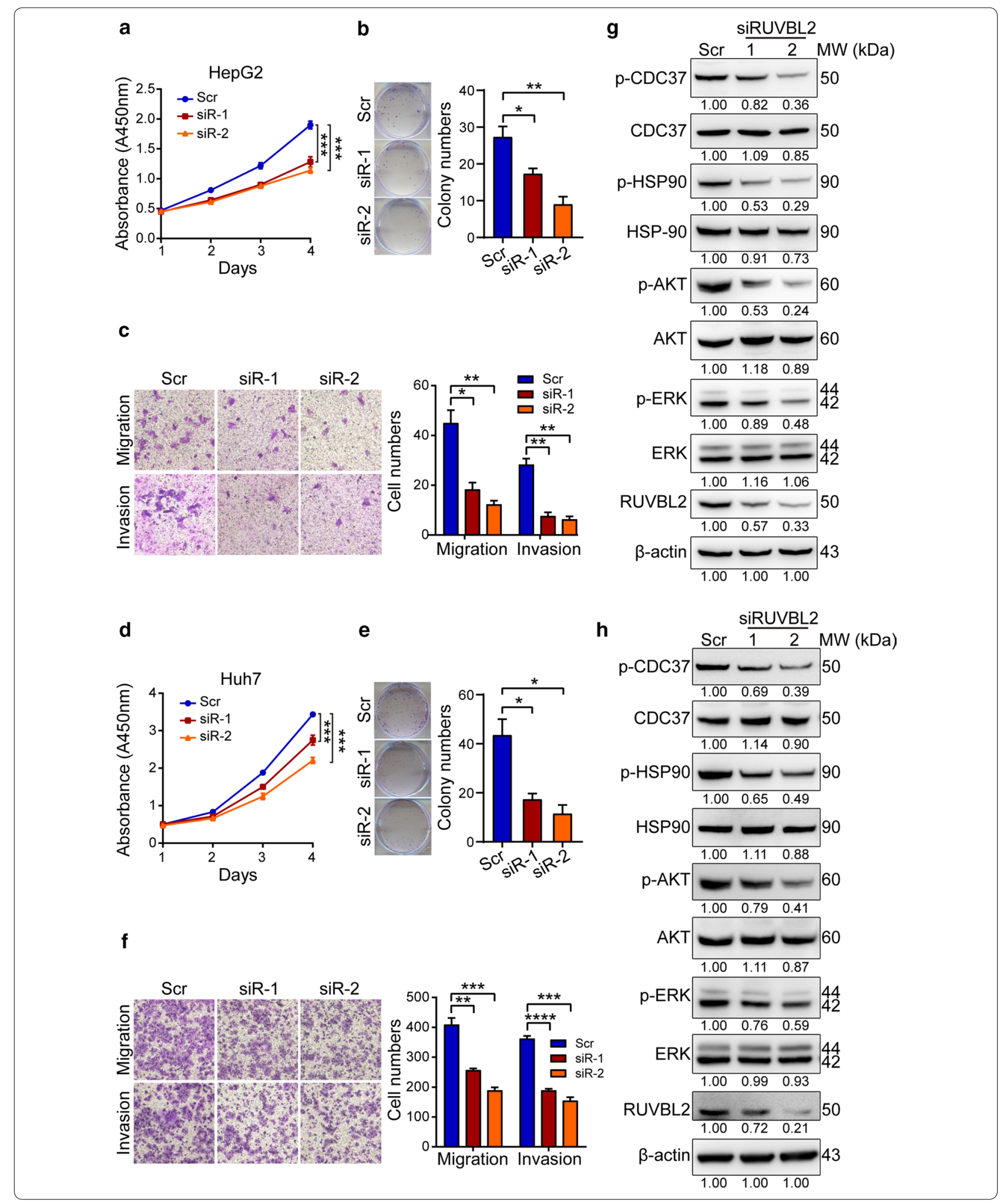


assessed, it was found that positive nuclear and cytoplasmic expression had different clinical and prognostic significance. It appears that patients with a cirrhotic background had decreased levels of nuclear RUVBL2, while poorly differentiated tumors showed dramatically increased levels of cytoplasmic RUVBL2. In addition, nuclear and cytoplasmic RUVBL2 independently indicated a worse overall survival and recurrence-free survival, respectively. According to the known functions of RUVBL2, nuclear forms might regulate DNA replication, chromatin remodeling, biogenesis of small nucleolar ribonucleoprotein (RNP) and small nuclear RNP, assembly of the telomerase complex, transcriptional regulation and DNA damage repair $[8,29]$. Moreover, cytoplasmic RUVBL2 can interact with phosphatidylinositol 3-kinaserelated protein kinase (PIKK) signaling family proteins to sense cellular nutrients and energy levels and to modulate the nonsense-mediated decay of mRNAs [30, 31]. RUVBL2 in the mitochondrion binds to mitochondrial DNA polymerase gamma (POLG) to participate in mitochondrial biogenesis [32]. Therefore, cytoplasmic RUVBL2 protein may play different roles from its nuclear forms in carcinogenesis; however, its cytoplasmic functions so far were not yet determined.

Furthermore, we found that high levels of RUVBL2 facilitated HCC cell proliferation, survival, migration and invasion. Previous studies reported that HSP90 can form complexes with RUVBL2 [31]; thus, we investigated the effect of RUVBL2 knockdown on HSP90-CDC37 complexes and the downstream pathways in HepG2 and Huh7 cells. We found that RUVBL2 depletion significantly attenuated the phosphorylation of HSP90, CDC37, ERK and AKT proteins (Fig. 5g, h). AKT is known as a HSP90 client kinase [33, 34], and CDC37 can stabilize ERK and AKT kinase activities in numerous cancer cells [34-36]. Therefore, these results demonstrated that RUVBL2 contributes to hepatocarcinogenesis via HSP90CDC37, AKT and ERK pathways.

\section{Conclusions}

We systematically investigated the mRNA and protein expression characteristics of RUVBL2 in HCC in a relatively large number of clinical samples. The results showed that promoter hypomethylation, copy number gain, $M Y C$ amplification and CTNNB1 mutation were responsible for the overexpression of RUVBL2. The high levels of RUVBL2 promote tumorigenesis through activating HSP90-CDC37, AKT and ERK pathways. RUVBL2 protein was distributed to the nucleus and the cytoplasm of malignant hepatocytes, and its different localization indicated distinct clinical and prognostic features. Therefore, high levels of nuclear and cytoplasmic RUVBL2 could be used as independent prognostic factor for overall survival and recurrence-free survival in HCC patients, respectively. RUVBL2 may also be a promising target for HCC prevention and treatment.

\section{Supplementary information}

Supplementary information accompanies this paper at https://doi. org/10.1186/s12935-019-0974-z.

Additional file 1: Figure S1. RUVBL2 mRNA expression according to the viruses infection status in Asian (A) and Caucasian (B) liver cancer samples from TCGA. Figure S2. The correlation of RUVBL2 and CTNNB1 mRNA in liver cancer patients with wild-type CTNNB1 (A) and mutant CTNNB1 (B).

\section{Authors' contributions}

TY: Performed the experiments, acquired and analyzed patient data; FL, JG and HL: Performed the experiments and collected the data; JC: Conceptualization; XZ: Study concept and design, funding acquisition; YS: Study concept and design, analysis of data, funding acquisition and drafting of the manuscript. All authors read and approved the final manuscript.

\section{Funding}

This work was supported by grants from the National Natural Science Foundation of China (Grant No. 81572840 and 81572365), Non-profit Central Research Institute Fund of CAMS (Grant No. 2018RC310011), National Key R \& D

Program (Grant No. 2017YFC0906601 and 2018YFC1313101), State Key Project for Infectious Diseases (Grant No. 2017ZX10203205-003) and CAMS Innovation Fund for Medical Sciences (Grant No. 2016-I2M-1-001, 2017-I2M-3-005 and 2019-12M-1-003).

\section{Availability of data and materials}

Please contact the authors for data request.

Consent for publication

All authors approved publication of the manuscript.

\section{Competing interests}

The authors declare that they have no competing interests.

\section{Author details}

${ }^{1}$ Department of Anesthesiology, National Cancer Center/National Clinical Research Center for Cancer/Cancer Hospital, Chinese Academy of Medical Science \& Peking Union Medical College, Beijing 100021, China. ${ }^{2}$ State Key Laboratory of Molecular Oncology, National Cancer Center/National Clinical Research Center for Cancer/Cancer Hospital, Chinese Academy of Medical Sciences \& Peking Union Medical College, 17 Panjiayuan Nanli, Chaoyang District, Beijing 100021, China. ${ }^{3}$ Department of Pathology, National Cancer Center/National Clinical Research Center for Cancer/Cancer Hospital, Chinese Academy of Medical Science \& Peking Union Medical College, Beijing 100021, China. ${ }^{4}$ Department of Hepatobiliary Surgery, National Cancer Center/ National Clinical Research Center for Cancer/Cancer Hospital, Chinese Academy of Medical Science \& Peking Union Medical College, Beijing 100021, China.

Received: 25 June 2019 Accepted: 23 September 2019

Published online: 27 September 2019

\section{References}

1. Bray F, Ferlay J, Soerjomataram I, Siegel RL, Torre LA, Jemal A. Global cancer statistics 2018: GLOBOCAN estimates of incidence and mortality worldwide for 36 cancers in 185 countries. CA Cancer J Clin. 2018;68(6):394-424

2. Chen W, Zheng R, Baade PD, Zhang S, Zeng H, Bray F, Jemal A, Yu XQ, He J. Cancer statistics in China, 2015. CA Cancer J Clin. 2016;66(2):115-32.

3. Zeng H, Chen W, Zheng R, Zhang S, Ji JS, Zou X, Xia C, Sun K, Yang Z, Li $\mathrm{H}$, et al. Changing cancer survival in China during 2003-15: a pooled 
analysis of 17 population-based cancer registries. Lancet Glob Health. 2018;6(5):e555-67.

4. Llovet JM, Zucman-Rossi J, Pikarsky E, Sangro B, Schwartz M, Sherman M, Gores G. Hepatocellular carcinoma. Nat Rev Dis Primers. 2016;2:16018.

5. Cancer Genome Atlas Research Network. Comprehensive and integrative genomic characterization of hepatocellular carcinoma. Cell. 2017;169(7):1327-1341.e1323.

6. Grigoletto A, Lestienne P, Rosenbaum J. The multifaceted proteins Reptin and Pontin as major players in cancer. Biochim Biophys Acta. 2011;1815(2):147-57.

7. Munoz-Hernandez H, Pal M, Rodriguez CF, Fernandez-Leiro R, Prodromou C, Pearl LH, Llorca O. Structural mechanism for regulation of the AAAATPases RUVBL1-RUVBL2 in the R2TP Co-chaperone revealed by cryo-EM. Sci Adv. 2019;5(5):eaaw1616.

8. Mao YQ, Houry WA. The role of pontin and reptin in cellular physiology and cancer etiology. Front Mol Biosci. 2017;4:58.

9. Rosenbaum J, Baek SH, Dutta A, Houry WA, Huber O, Hupp TR, Matias PM. The emergence of the conserved AAA+ ATPases Pontin and Reptin on the signaling landscape. Sci Signal. 2013;6(266):mr1.

10. Qiu H, Gao Y, Mao D. Reptin physically interacts with $p 65$ and represses NF-kappaB activation. FEBS Lett. 2015;589(15):1951-7.

11. Maslon MM, Hrstka R, Vojtesek B, Hupp TR. A divergent substrate-binding loop within the pro-oncogenic protein anterior gradient- 2 forms a docking site for Reptin. J Mol Biol. 2010;404(3):418-38.

12. Cho SG, Bhoumik A, Broday L, Ivanov V, Rosenstein B, Ronai Z. TIP49b, a regulator of activating transcription factor 2 response to stress and DNA damage. Mol Cell Biol. 2001;21(24):8398-413.

13. Bauer A, Chauvet S, Huber O, Usseglio F, Rothbacher U, Aragnol D, Kemler $\mathrm{R}$, Pradel J. Pontin52 and reptin52 function as antagonistic regulators of beta-catenin signalling activity. EMBO J. 2000;19(22):6121-30.

14. Lee JS, Kim Y, Kim IS, Kim B, Choi HJ, Lee JM, Shin HJ, Kim JH, Kim JY, Seo $S B$, et al. Negative regulation of hypoxic responses via induced Reptin methylation. Mol Cell. 2010;39(1):71-85.

15. Armenteros-Monterroso E, Zhao L, Gasparoli L, Brooks T, Pearce K, Mansour MR, Martens JHA, de Boer J, Williams O. The AAA+ ATPase RUVBL2 is essential for the oncogenic function of c-MYB in acute myeloid leukemia. Leukemia. 2019. https://doi.org/10.1038/s41375-019-0495-8.

16. Rousseau B, Menard L, Haurie V, Taras D, Blanc JF, Moreau-Gaudry F, Metzler $\mathrm{P}$, Hugues M, Boyault S, Lemiere S, et al. Overexpression and role of the ATPase and putative DNA helicase RuvB-like 2 in human hepatocelIular carcinoma. Hepatology. 2007;46(4):1108-18.

17. Li W, Zeng J, Li Q, Zhao L, Liu T, Bjorkholm M, Jia J, Xu D. Reptin is required for the transcription of telomerase reverse transcriptase and overexpressed in gastric cancer. Mol Cancer. 2010;9:132.

18. Ren J, Li W, Liu H, Yan L, Jiao W, Li D, Tang Y, Gu G, Xu Z. Overexpression of reptin in renal cell carcinoma contributes to tumor malignancies and its inhibition triggers senescence of cancer cells. Urol Oncol. 2013;31(7):1358-66.

19. Flavin P, Redmond A, McBryan J, Cocchiglia S, Tibbitts P, Fahy-Browne P, Kay E, Treumann A, Perrem K, Mcllroy M, et al. RuvBl2 cooperates with Ets2 to transcriptionally regulate hTERT in colon cancer. FEBS Lett. 2011;585(15):2537-44.

20. Mikesch JH, Hartmann W, Angenendt L, Huber O, Schliemann C, Arteaga MF, Wardelmann E, Rudack C, Berdel WE, Stenner M, et al. AAA+ ATPases Reptin and Pontin as potential diagnostic and prognostic biomarkers in salivary gland cancer-a short report. Cell Oncol. 2018;41(4):455-62.

21. Menard L, Taras D, Grigoletto A, Haurie V, Nicou A, Dugot-Senant N, Costet P, Rousseau B, Rosenbaum J. In vivo silencing of Reptin blocks the progression of human hepatocellular carcinoma in xenografts and is associated with replicative senescence. J Hepatol. 2010;52(5):681-9.

22. Raymond AA, Benhamouche S, Neaud V, Di Martino J, Javary J, Rosenbaum J. Reptin regulates DNA double strand breaks repair in human hepatocellular carcinoma. PLoS ONE. 2015;10(4):e0123333.

23. Breig O, Yates M, Neaud V, Couchy G, Grigoletto A, Lucchesi C, Prox J, Zucman-Rossi J, Becker-Pauly C, Rosenbaum J. Metalloproteinase meprin alpha regulates migration and invasion of human hepatocarcinoma cells and is a mediator of the oncoprotein Reptin. Oncotarget. 2017:8(5):7839-51.

24. Javary J, Allain-Courtois N, Saucisse N, Costet P, Heraud C, Benhamed F, Pierre R, Bure C, Pallares-Lupon N, Do Cruzeiro M, et al. Liver Reptin/ RUVBL2 controls glucose and lipid metabolism with opposite actions on mTORC1 and mTORC2 signalling. Gut. 2018;67(12):2192-203.

25. Raymond AA, Javary J, Breig O, Neaud V, Rosenbaum J. Reptin regulates insulin-stimulated Akt phosphorylation in hepatocellular carcinoma via the regulation of SHP-1/PTPN6. Cell Biochem Funct. 2017;35(6):289-95.

26. Hao XP, Pretlow TG, Rao JS, Pretlow TP. Beta-catenin expression is altered in human colonic aberrant crypt foci. Cancer Res. 2001;61(22):8085-8.

27. Wood MA, McMahon SB, Cole MD. An ATPase/helicase complex is an essential cofactor for oncogenic transformation by c-Myc. Mol Cell. 2000;5(2):321-30

28. Menssen A, Hermeking H. Characterization of the c-MYC-regulated transcriptome by SAGE: identification and analysis of C-MYC target genes. Proc Natl Acad Sci USA. 2002;99(9):6274-9.

29. Cloutier P, Poitras C, Durand M, Hekmat O, Fiola-Masson E, Bouchard A, Faubert D, Chabot B, Coulombe B. R2TP/Prefoldin-like component RUVBL1/RUVBL2 directly interacts with ZNHIT2 to regulate assembly of U5 small nuclear ribonucleoprotein. Nat Commun. 2017:8:15615.

30. Izumi N, Yamashita A, Iwamatsu A, Kurata R, Nakamura H, Saari B, Hirano $\mathrm{H}$, Anderson P, Ohno S. AAA+ proteins RUVBL1 and RUVBL2 coordinate PIKK activity and function in nonsense-mediated mRNA decay. Sci Signal. 2010;3(116):ra27.

31. Izumi N, Yamashita A, Hirano H, Ohno S. Heat shock protein 90 regulates phosphatidylinositol 3-kinase-related protein kinase family proteins together with the RUVBL1/2 and Tel2-containing co-factor complex. Cancer Sci. 2012;103(1):50-7.

32. Liyanage SU, Coyaud E, Laurent EM, Hurren R, Maclean N, Wood SR, Kazak L, Shamas-Din A, Holt I, Raught B, et al. Characterizing the mitochondrial DNA polymerase gamma interactome by BiolD identifies Ruvbl2 localizes to the mitochondria. Mitochondrion. 2017;32:31-5.

33. Sato S, Fujita N, Tsuruo T. Modulation of Akt kinase activity by binding to Hsp90. Proc Natl Acad Sci USA. 2000;97(20):10832-7.

34. Citri A, Harari D, Shohat G, Ramakrishnan P, Gan J, Lavi S, Eisenstein M, Kimchi A, Wallach D, Pietrokovski S, et al. Hsp90 recognizes a common surface on client kinases. J Biol Chem. 2006;281 (20):14361-9.

35. Gray PJ Jr, Stevenson MA, Calderwood SK. Targeting Cdc37 inhibits multiple signaling pathways and induces growth arrest in prostate cancer cells. Cancer Res. 2007;67(24):11942-50.

36. Piatelli MJ, Doughty C, Chiles TC. Requirement for a hsp90 chaperone-dependent MEK1/2-ERK pathway for B cell antigen receptorinduced cyclin D2 expression in mature B lymphocytes. J Biol Chem. 2002;277(14):12144-50.

\section{Publisher's Note}

Springer Nature remains neutral with regard to jurisdictional claims in published maps and institutional affiliations. 